\title{
Circular RNA ciRS-7 affects the propagation of Cryptosporidium parvum in HCT-8 cells by sponging miR-1270 to activate the NF-KB signaling pathway
}

\author{
Yan-Ling Yin ${ }^{1}$, Ting-Li Liư ${ }^{2}$, Qian Yao ${ }^{1}$, Yu-Xin Wang ${ }^{1}$, Xue-Mei Wu' ${ }^{1}$ Xue-Ting Wang ${ }^{1}$, Xin Yang ${ }^{1}$, \\ Jun-Ke Song ${ }^{1}$ and Guang-Hui Zhao ${ }^{1 *}$
}

\begin{abstract}
Background: Cryptosporidium is an important zoonotic pathogen responsible for severe enteric diseases in humans and animals. However, the molecular mechanisms underlying host and Cryptosporidium interactions are still not clear.

Methods: To study the roles of circRNAs in host cells during Cryptosporidium infection, the expression profiles of circRNAs in HCT-8 cells infected with C. parvum were investigated using a microarray assay, and the regulatory role of a significantly upregulated circRNA, ciRS-7, was investigated during C. parvum infection.

Results: C. parvum infection caused notable alterations in the expression profiles of circRNAs in HCT-8 cells, and a total of 178 (including 128 up- and 50 downregulated) circRNAs were significantly differentially expressed following C. parvum infection. Among them, ciRS-7 was significantly upregulated and regulated the NF-KB signaling pathway by sponging miR-1270 during C. parvum infection. Furthermore, the ciRS-7/miR-1270/relA axis markedly affected the propagation of C. parvum in HCT-8 cells.

Conclusions: Our results revealed that ciRS-7 would promote C. parvum propagation by regulating the miR1270/relA axis and affecting the NF-KB pathway. To the best of our knowledge, this is the first study to investigate the role of circRNA during Cryptosporidium infection, and the findings provide a novel view for implementing control strategies against Cryptosporidium infection.
\end{abstract}

Keywords: C. parvum, CircRNAs, CiRS-7/miR-1270/Re/A axis, Propagation, NF-kB signaling pathway

\section{Background}

Cryptosporidiosis, caused by the protozoan zoonotic parasite Cryptosporidium, is a ubiquitous gastrointestinal illness of humans and many domestic and wild animals. Asymptomatic to severe clinical outcomes can occur because of Cryptosporidium infection, and diarrhea is the

*Correspondence: zgh083@nwsuaf.edu.cn

${ }^{1}$ Department of Parasitology, College of Veterinary Medicine, Northwest A\&F University, Yangling, China

Full list of author information is available at the end of the article most common sequela. Cryptosporidium has been listed as the fifth leading cause of diarrhea in children $<5$ years of age by the Global Burden of Disease Study (GBD) 2016 and has been identified as the second leading cause of diarrhea-associated mortality in children of this age by the Global Burden of Disease Study (GBD) 2015 [1, 2]. Apart from diarrhea, malabsorption and growth impairment are also found in children in less developed countries and impoverished nations due to chronic infection with Cryptosporidium [3, 4]. Furthermore, cryptosporidiosis is an important food- and waterborne disease that

c) The Author(s) 2021. This article is licensed under a Creative Commons Attribution 4.0 International License, which permits use, sharing, adaptation, distribution and reproduction in any medium or format, as long as you give appropriate credit to the original author(s) and the source, provide a link to the Creative Commons licence, and indicate if changes were made. The images or other third party material in this article are included in the article's Creative Commons licence, unless indicated otherwise in a credit line to the material. If material is not included in the article's Creative Commons licence and your intended use is not permitted by statutory regulation or exceeds the permitted use, you will need to obtain permission directly from the copyright holder. To view a copy of this licence, visit http://creativeco mmons.org/licenses/by/4.0/. The Creative Commons Public Domain Dedication waiver (http://creativecommons.org/publicdomain/ zero/1.0/) applies to the data made available in this article, unless otherwise stated in a credit line to the data. 
is responsible for $>8$ million cases of foodborne illness annually with $>524$ waterborne outbreaks documented from the start of the last century to 2016 [2, 4-6]. The serious consequences of cryptosporidiosis are underestimated and underreported owing to commonly asymptomatic carriage in immunocompetent hosts, insufficient attention, and the unavailability of perfect diagnostic procedures for this important disease worldwide. More significantly, vaccines or effective therapeutics to control cryptosporidiosis are still insurmountable challenges. Thus far, only one drug, nitazoxanide, has been proven to treat cryptosporidiosis [7]. However, this drug is only $56-96 \%$ effective in immunocompetent hosts, and no efficacy is found in immune-compromised individuals, especially in patients with advanced AIDS [7]. Considering that the severity of cryptosporidiosis is closely associated with host status, especially immunity $[7,8]$, thoroughly understanding the interaction between hosts and Cryptosporidium is essential to develop effectively well-directed control strategies against cryptosporidiosis.

In recent years, noncoding RNAs (ncRNAs), comprising $95 \%$ of the total amount of RNA and transcribed from what were previously considered "junk DNA" sequences, have emerged as novel functional molecules or biomarkers in the progression and processes of a variety of diseases $[9,10]$. Among these, circular RNA (circRNA), unlike common linear RNAs, is a covalently closed-ring molecule without the $5^{\prime}-3^{\prime}$ polar covalently closed loop structure or the polycyclic adenylate tail [11, 12]. This unique RNA transcript is resistant to RNase R, and ongoing studies have shown that it can act as a novel key regulator of gene expression [11]. Next-generation RNA sequencing (RNA-seq) of nonpolyadenylated transcriptomes has uncovered $>10,000$ different circRNAs in metazoans [11], and aberrant expression of host circRNAs was induced by infections of various pathogens, including bacteria (e.g. Mycobacterium tuberculosis, Chlamydia trachomatis), viruses (e.g. porcine endemic diarrhea virus, transmissible gastroenteritis virus), and parasites (e.g. Nosema ceranae, Toxoplasma gondii, Eimeria necatrix) [13-18]. Using the RNA-seq technique without RNase R treatment, our group found 104 significantly differentially expressed circRNAs in tracheal tissues of chickens experimentally infected with C. baileyi. Pathway enrichment of these differential circRNAs identified several pathways (e.g. amino sugar and nucleotide sugar metabolism, tight junction, and glycerolipid metabolism) associated with cryptosporidiosis [19], suggesting the potentially important roles of these circRNAs during Cryptosporidium infection. However, a small number of circRNAs were identified by the RNA-seq technique used in this study, and fake circRNAs were produced by using this technique. Furthermore, the functional mechanisms of circRNAs during Cryptosporidium infection were not revealed in this work. To date, $>40$ valid Cryptosporidium species have been recognized [4]. Among them, $C$. parvum is the most common zoonotic species infecting humans and many animals $[20,21]$ and is responsible for the majority of foodborne outbreaks [4]. In the present study, the expression profiles of circRNAs were investigated in HCT-8 cells infected with C. parvum using a microarray assay with RNA samples after treatment with RNase $R$, and the role and mechanism of a significantly upregulated circRNA, ciRS-7, were preliminarily studied.

\section{Methods}

\section{Cells, parasites, and in vitro infection model}

The human ileocecal adenocarcinoma (HCT-8) cell line was obtained from JENNIO Biological Technology (Guangzhou, China) and cultured in RPMI 1640 medium supplemented with $10 \%$ fetal bovine serum at $37{ }^{\circ} \mathrm{C}$ with $5 \% \mathrm{CO}_{2}$. C. parvum oocysts were initially collected from neonatal dairy calves with clinical symptoms of diarrhea in Yangling of Shaanxi Province, northwestern China, identified as the C. parvum IIdA19G1 subtype using molecular tools targeting the $18 \mathrm{~S}$ rRNA gene and $60 \mathrm{kDa}$ glycoprotein ( $g p 60)$ gene (data not shown) and propagated in neonatal dairy calves under conditions with no specific pathogens. The C. parvum oocysts were purified by Sheather's sugar flotation and cesium chloride density gradient centrifugation, resuspended in phosphatebuffered saline solution (PBS) supplemented with $100 \mathrm{U} /$ $\mathrm{ml}$ penicillin, $100 \mu \mathrm{g} / \mathrm{ml}$ streptomycin, and $0.25 \mu \mathrm{g} / \mathrm{ml}$ amphotericin $\mathrm{B}$ solutions, and stored at $4{ }^{\circ} \mathrm{C}$. An in vitro infection model of $C$. parvum was established according to our previous study [21], using an infection ratio of 2-10:1 between oocysts and HCT-8 cells.

\section{Total RNA extraction and circRNA microarray analysis}

Total RNA was isolated using the TRIzol reagent (Invitrogen, Gaithersburg, MD, USA) and purified by using a NucleoSpin ${ }^{\circledR}$ RNA clean-up kit (MACHEREY-NAGEL, Dueren, Germany) according to the manufacturer's instructions. The purity and integrity of the total RNA were determined using a ND-1000 Spectrophotometer (Thermo Fisher Scientific Inc., Wilmington, DE, USA) and $1 \%$ agarose gel electrophoresis, respectively. The qualified total RNA was treated with RNase R (Epicentre, Madison, WI, USA) to remove linear RNA and then transcribed into double-strand cDNA using an Ambion ${ }^{\circledR}$ WT Expression Kit (Thermo Fisher Scientific Inc., Wilmington, DE, USA). The second strand of cDNA was used as the template to synthesize the cRNA, which was then purified by an RNA purification column to remove the salt, enzyme, and other reagents in the reaction and transcribed into cDNA utilizing random primers. The 
transcribed cDNA was labeled with Cy3-dCTP and hybridized with the circRNA Human Gene Expression Microarray Array V2.0 $(4 \times 180 \mathrm{~K})$ (CapitalBio Technology Corp., Beijing, China). The hybridization arrays were washed and scanned by using an Agilent Scanner (G2565CA), and Agilent Feature Extraction v10.7 software was used to analyze and extract raw data from scanned images. The extracted data were normalized and processed by using Agilent GeneSpring software. The differentially expressed circRNAs during C. parvum infection showing statistical significance were filtered by fold change $>2.0$ and $P$-value $\leq 0.05$.

\section{Quantitative real-time polymerase chain reaction (qRT-PCR)}

Total RNA samples were isolated using TRIzol reagent (Beijing CoWin Biotech Co., Ltd., Beijing, China) and then transcribed into cDNA for RNA and miRNA verification by using the Primescript ${ }^{\mathrm{TM}}$ reagent kit with gDNA Eraser (Takara Biomedical Technology, Dalian, China) and the Mir-X miRNA First-Strand Synthesis Kit (Takara Biomedical Technology, Dalian, China) according to the manufacturer's instructions. qRT-PCRs were performed by using SBRY Green PCR Master Mix (Beijing CoWin Biotech Co., Ltd., Beijing, China), with gapdh and $u 6$ used as internal references for RNAs and miRNAs, respectively. Experiments were carried out in triplicate, and the relative expression of each RNA was calculated using the $2^{-\Delta \Delta C t}$ method. The primer sequences used in the present study are listed in Additional file 1: Table S1.

\section{Cell transfection}

Small interfering RNA (siRNA) against ciRS-7 (si-ciRS-7) and scramble RNA (si-control) was purchased from RiboBio Co., Ltd. (Guangzhou, China), and the ciRS-7 overexpression vector was constructed by using the pcDNA3.1 $(+)$ plasmid (Invitrogen, Gaithersburg, MD, USA). miR-1270 mimics and inhibitors with their negative controls were obtained from GenePharma (Shanghai, China). For transfection, Lipofectamine 2000 reagent (Invitrogen, Gaithersburg, MD, USA) was used following the manufacturer's protocols. The sequences of siRNA and miRNA mimics and inhibitors are presented in Additional file 2: Table S2.

\section{Western blotting}

The protein samples were extracted by RIPA lysis buffer (Beijing Applygen Technologies Co., Ltd., Beijing, China) supplemented with protease and phosphatase inhibitors (Beijing Solarbio Science \& Technology Co., Ltd., Beijing, China) and denatured in boiling water for $10 \mathrm{~min}$. The denatured proteins were separated by sodium dodecyl sulfate polyacrylamide gel electrophoresis (SDS-PAGE) and transferred to polyvinylidene difluoride (PVDF) membranes (Millipore, Billerica, USA). The membranes were blocked in PBST $(0.05 \%$ Tween-20) solution containing 5\% nonfat milk for $1 \mathrm{~h}$ to remove nonspecific binding. Following overnight incubation with the primary antibodies anti-RELA (1:1000, ABclonal, Wuhan, China) or anti-GAPDH (1:500,000, ABclonal, Wuhan, China) at $4{ }^{\circ} \mathrm{C}$, the membranes were incubated with a horseradish peroxidase (HRP)-conjugated secondary antibody (1:2000, Shanghai Sangon Biotech, Shanghai, China) at room temperature for $1 \mathrm{~h}$. An enhanced chemiluminescence (ECL) system was applied to visualize protein bands, and ImageJ software was used to quantify protein expression levels.

\section{Dual luciferase reporter assay}

The plasmids of wild-type ciRS-7 (ciRS-7-WT) and relA (relA-WT) and mutant ciRS-7 (ciRS-7-MUT) and relA (relA-MUT) were designed and inserted into pmirGLO vectors (Promega, Madison, WI, USA). Each constructed plasmid was cotransfected with miR-1270 mimics or control mimics into HCT-8 cells by using Lipofectamine 2000 reagent (Invitrogen, Gaithersburg, MD, USA). Luciferase activities were measured after $48 \mathrm{~h}$ of incubation by using a multimode reader assay (Spark, Tecan, Männedorf, Switzerland).

\section{Statistical analysis}

Results were presented as the mean $\pm \mathrm{SD}$. Data from different groups were compared by using one-way ANOVA. All statistical analyses were processed using GraphPad PRISM 6.07 software (San Diego, CA, USA) or IBM SPSS statistics version 22.0 software (Armonk, NY, USA). A $P$-value $<0.05$ was considered statistically significant.

\section{Results}

\section{C. parvum infection induced significantly aberrant} expression of circRNA profiles in HCT-8 cells

Following Cryptosporidium infection for $24 \mathrm{~h}$, a total of 178 (including 128 up- and 50 downregulated) circRNAs were found to be significantly differentially expressed (DE) in HCT-8 cells with a fold change $>2.0$ and $P \leq 0.05$ (Fig. 1a, b and Additional file 3: Table S3). GO and KEGG pathway analysis of genes producing DE circRNAs indicated that these DE circRNAs were involved in several important biological processes (e.g. leukocyte chemotaxis, chemotaxis, and cellular response to epinephrine stimulus), molecular functions (e.g. adrenergic receptor binding, adenyl ribonucleotide binding, and adenyl nucleotide binding) (Additional file 4: Table S4 and Additional file 6: Figure S1), and vital signaling pathways (e.g. ECM-receptor interaction, focal adhesion, and purine metabolism) (Additional file 5: Table S5 and 

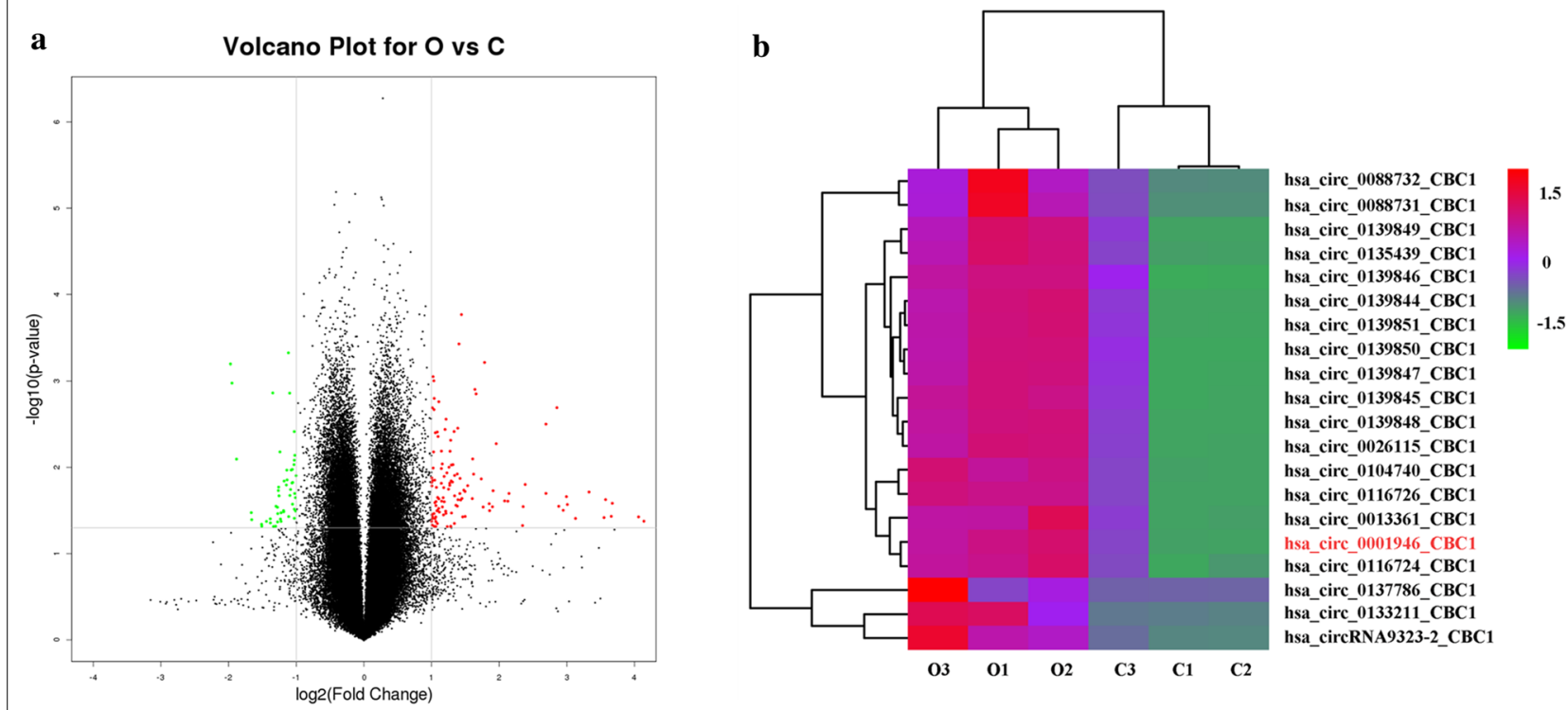

Fig. 1 Microarray analysis of differentially expressed circRNAs in HCT-8 cells infected with Cryptosporidium parvum IIdA19G1 subtype. a Volcano plots showed differently expressed circRNAs in response to C. parvum infection. Significantly differentially expressed circRNAs with a fold change $>2.0$ and $P \leq 0.05$ are shown in red (upregulated) or green (downregulated) dots. $\mathbf{b}$ Heat maps presented the top 20 significantly upregulated circRNAs in HCT-8 cells in response to C. parvum infection

Additional file 7: Figure S2) during host-Cryptosporidium interaction.

\section{The expression of ciRS-7 was induced by C. parvum infection in HCT-8 cells}

Among 128 significantly upregulated circRNAs, a previously well-studied circRNA, hsa_circ_0001946_CBC1 (ciRS-7, also known as CDR1as), was remarkably upregulated by nearly eightfold following $C$. parvum infection for $24 \mathrm{~h}$ in microarray analysis (Additional file 3: Table S3). qRT-PCR confirmed the microarray results and found continuously increased expression of ciRS-7 in HCT-8 cells from 12-h post-infection (pi) to $48 \mathrm{hpi}$ (Fig. 2a), suggesting the potential role of this circRNA during $C$. parvum infection.

\section{CiRS-7 acted as a sponge of miR-1270 in HCT-8 cells following $C$. parvum infection}

Considering the involvement of ciRS-7 in many physiological or pathological processes via binding to specific miRNAs [22-24], we predicted 22 potential miRNA targets of ciRS-7 by using starBase v2.0 [25]. By constructing ciRS-7-miRNA networks (Additional file 8: Figure S3) with Cytoscape software [26], we identified a miRNA, miR-1270, which was one of the most highly matched miRNAs of ciRS-7 [27] and acted as a sponge to regulate the progression of ovarian cancer [28], bladder cancer [29], and hepatocellular carcinoma [27]. In the present study, the expression of miR-1270 was significantly decreased in HCT-8 cells infected with C. parvum at 24 hpi (Fig. 2b), and the dual-luciferase reporter assay showed that cotransfection of pmirGLO-ciRS-7-WT luciferase reporter vector with miR-1270 mimics, but not pmirGLO-ciRS-7-MUT vector, markedly reduced the ciRS-7-regulated luciferase activity in HCT-8 cells (Fig. 2c). Furthermore, overexpression of ciRS-7 conspicuously decreased miR-1270 expression levels in C. parvum-infected HCT-8 cells, while knockdown of ciRS-7 using siRNA targeting ciRS-7 had the reverse effect (Fig. 2b and Additional file 9: Figure S4). These results demonstrated that ciRS-7 might sponge miR-1270 in HCT-8 cells in response to C. parvum infection.

\section{RelA was a direct target of miR-1270 in HCT-8 cells following C. parvum infection}

It is well known that miRNAs can regulate various biological processes through perfect or partial base pairing of the $3^{\prime}$-UTR regions of the target mRNAs [30]. To better understand the potential function of miR-1270 in HCT-8 cells following C. parvum infection, relA (also known as $p 65$ ), a subunit of the NF- $\mathrm{kB}$ signaling pathway which was proven to be a key pathway to prevent epithelial cell apoptosis, thus benefiting parasite propagation [31], was predicted to be a potential target of miR-1270 by using the starBase v2.0 database. In our study, the mRNA level of relA was significantly upregulated during C. parvum infection from 12 to $48 \mathrm{hpi}$ (Fig. 3a), and the protein level was remarkably increased in HCT-8 cells 


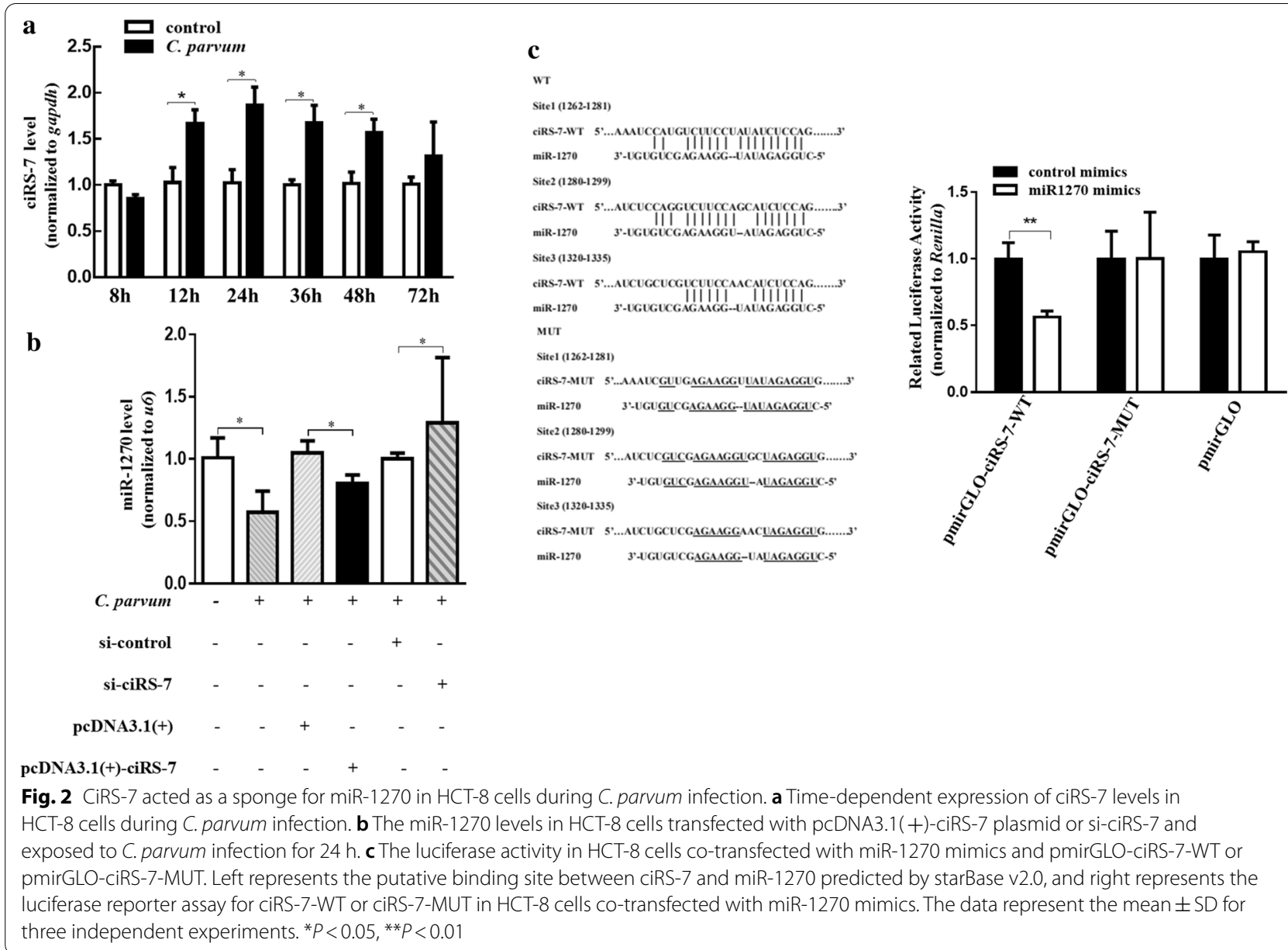

exposed to C. parvum for 24 h (Fig. 3b). A dual-luciferase reporter assay found that the luciferase activity of the pmirGLO-relA-WT reporter vector was significantly decreased by miR-1270 mimics (Fig. 3c). Moreover, both qRT-PCR (Fig. 3d) and western blot (Fig. 3b) assays showed that relA expression was decreased by miR-1270 mimics but increased by miR-1270 inhibitor in HCT- 8 cells infected with $C$. parvum. Thus, miR-1270 might directly regulate relA mRNA and RELA protein expression during C. parvum infection in HCT-8 cells.

\section{CiRS-7 regulated relA expression by sponging miR-1270 in HCT-8 cells following C. parvum infection}

CircRNAs act as competing endogenous RNAs (ceRNAs) to sponge miRNAs and consequently regulate the expression of the targeted mRNAs [32]. In the present study, we tested whether ciRS-7 modulated relA expression by adsorbing miR-1270 in HCT-8 cells following C. parvum infection. We found that the expression pattern of relA was similar to that of ciRS-7 and was also significantly increased from 12 to $48 \mathrm{hpi}$ (Fig. 3a). The upregulation of relA induced by $C$. parvum was attenuated by silencing of
ciRS-7 using siRNA (si-ciRS-7), while overexpression of ciRS-7 enhanced the effect of C. parvum on the relA gene (Fig. 4a, b). Cotransfection of pcDNA3.1(+)-ciRS-7 vector and miR-1270 mimics into HCT-8 cells showed that the upregulated effect of ciRS-7 on relA expression during C. parvum infection was reversed by $\mathrm{miR}-1270 \mathrm{mim}$ ics (Fig. 4c). These results indicated that ciRS-7 regulated relA by targeting miR-1270 during C. parvum infection.

\section{CiRS-7 stimulated the NF-KB pathway by sponging miR-1270 in HCT-8 cells following C. parvum infection} Given that ciRS-7 affected the expression of the subunit relA within the NF-kB signaling pathway by sponging miR-1270 during C. parvum infection, we asked whether the ciRS-7/miR-1270 axis regulated the NF- $\mathrm{kB}$ signaling pathway. To address this question, we investigated the expression of two important downstream molecules of the NF- $\mathrm{kB}$ signaling pathway, nos 2 and $c x c l 2$, which were both upregulated during C. parvum infection [33]. In our study, the expression of nos 2 and $c x c l 2$ was remarkably increased in HCT-8 cells infected with C. parvum at 24 hpi (Fig. 5). Overexpression of ciRS-7 markedly increased 


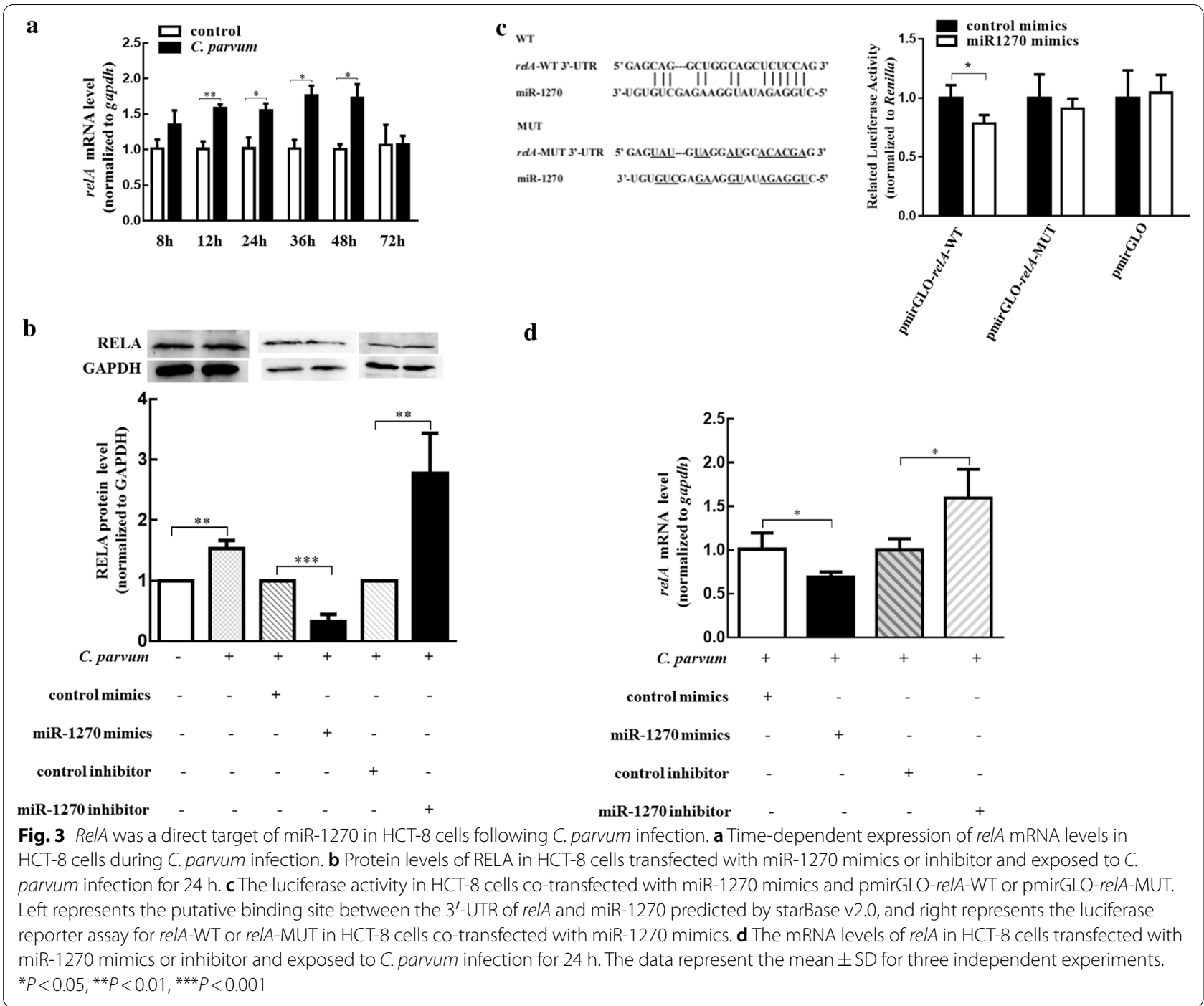

the expression of two genes in HCT-8 cells infected with C. parvum, while knockdown of ciRS-7 significantly decreased their expression (Fig. 5a, b). Notably, the effect of miR-1270 on the expression of nos 2 and $c x c l 2$ was exactly the opposite of that of ciRS-7 (Fig. 5c, d), and miR-1270 could attenuate the induction effect of ciRS-7 on $n o s 2$ and $c x c l 2$ (Fig. 6a, b), suggesting that ciRS-7 was implicated in activating the NF-kB pathway by interacting with miR-1270 during C. parvum infection.

\section{Upregulation of ciRS-7 promoted the propagation of $C$. parvum by regulating miR-1270 in HCT-8 cells}

Activation of the NF- $\mathrm{kB}$ signaling pathway significantly affects the propagation of $C$. parvum $[31,33]$. Therefore, we studied the role of the ciRS-7/miR-1270 axis in determining the infection burden of C. parvum in HCT-8 cells. The mRNA level of the $h s p 70$ gene of $C$. parvum was enhanced in HCT-8 cells transfected with pcDNA3.1(+)-ciRS-7 compared with control groups with pcDNA3.1 $(+)$ (Fig. 7a and Additional file 10: Figure S5), and significantly decreased expression of the hsp 70 gene of C. parvum was detected in HCT-8 cells transfected with si-ciRS-7 (Fig. 7a). On the other hand, miR-1270 mimics markedly decreased the expression level of the C. parvum hsp70 gene (Fig. 7b), but the opposite effect on the infection burden was detected in HCT-8 cells by knockdown of miR-1270 with an inhibitor (Fig. 7b). Furthermore, cotransfection assay showed that the upregulated effect of ciRS-7 on parasite burden was markedly suppressed by miR-1270 mimics (Fig. 7c), indicating that ciRS-7 promoted $C$. parvum propagation by regulating miR-1270 expression. 


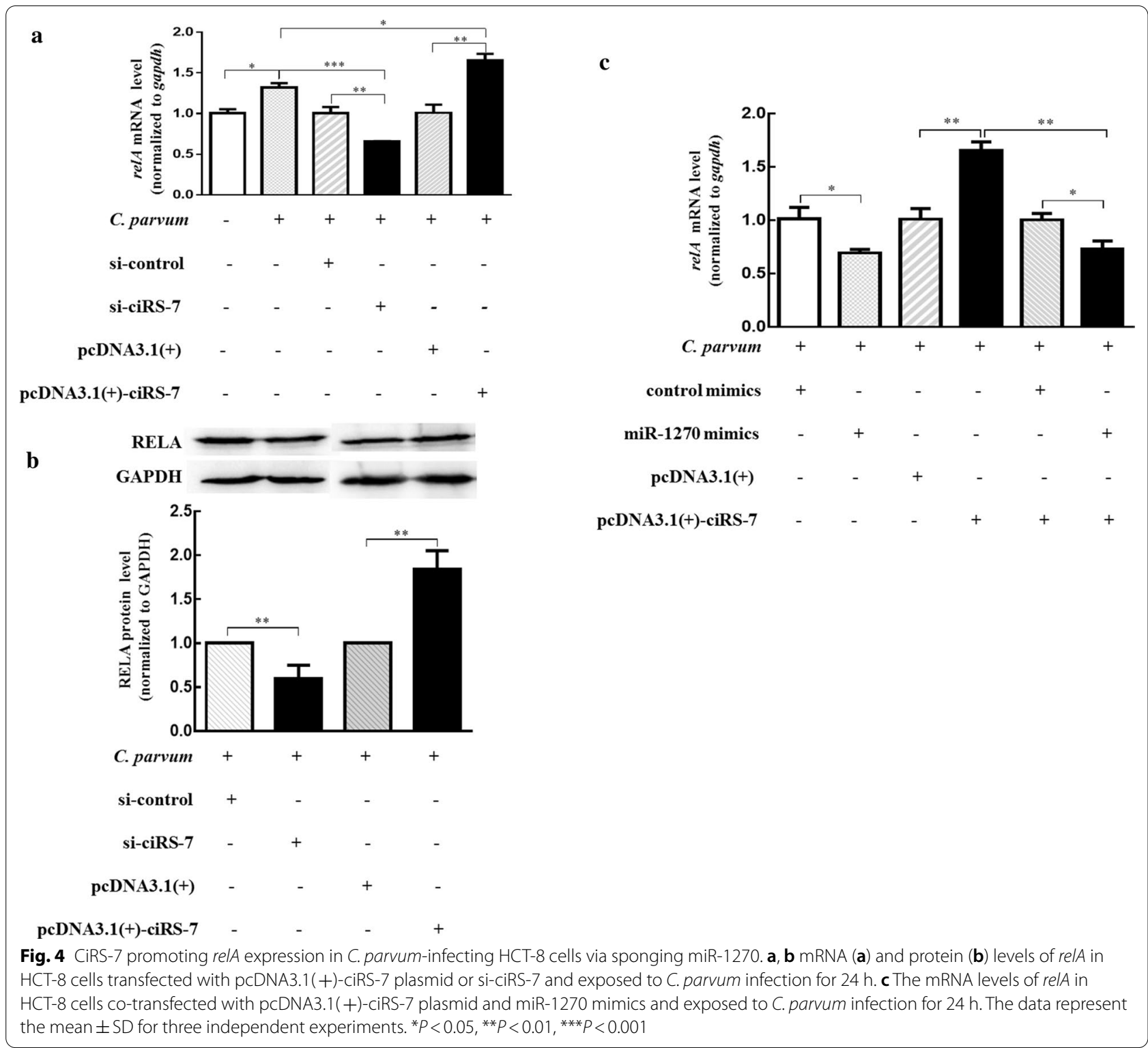

\section{Discussion}

In recent years, circRNAs, a novel RNA class, are being recognized to function in many kinds of physiological and pathological processes by regulating gene expression at the transcriptional and posttranscriptional levels [34-36]. Significantly aberrant expression of host circRNAs at the genome-wide level was also found during infections with a limited number of parasites [13, 18, 37], including Cryptosporidium [19]; however, the functions of these DE circRNAs are still unknown. In the present study, we performed microarray analysis of DE circRNAs in HCT-8 cells infected with the important zoonotic $C$. parvum and identified a total of 178 circRNAs (including 128 upregulated and 50 downregulated) that were abnormally expressed. Furthermore, we found that upregulated ciRS-7 promoted the in vitro propagation of Cryptosporidium by acting as a miRNA sponge.

ciRS-7, originating from cerebellar degenerationrelated protein 1 antisense transcript (CDR1AS), is the most well-known and widely studied circRNA [38, 39]. This circular ncRNA molecule serves as a regulator in many diseases, including many cancers (e.g. breast cancer, colorectal cancer, bladder cancer, esophageal cancer, gastric cancer, hepatocellular cancer, lung cancer, pancreatic cancer, ovarian cancer), gliomas, diabetes, myocardial infarction, atherosclerosis, osteoarthritis, pulmonary fibrosis, osteonecrosis of the femoral head, myocardial infarction, and neurodegenerative diseases 
$\mathbf{a}$

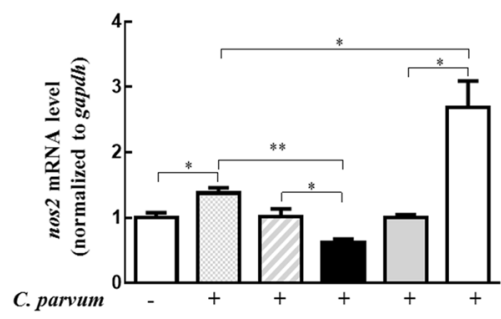

si-control

si-ciRS-7

pcDNA3.1 $(+)$

pcDNA3.1(+)-ciRS-7

c

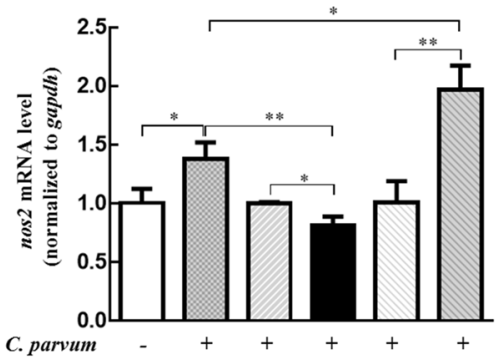

control mimics

miR-1270 mimic

control inhibitor

miR-1270 inhibitor

Fig. 5 The impact of ciRS-7/miR-1270 axis on the expression of NF-KB signal downstream molecules of nos2 (a, c) and cxcl2 (b, d) in HCT-8 cells following C. parvum infection. HCT-8 cells were transfected with pCDNA3.1(+)-ciRS-7 plasmid or si-ciRS-7 $(\mathbf{a}, \mathbf{b})$ and transfected with miR-1270 mimics or inhibitor $(\mathbf{c}, \mathbf{d})$, followed by exposing to C. parvum infection for $24 \mathrm{~h}$. The mRNA levels of nos $2(\mathbf{a}, \mathbf{c})$ and $c x c / 2(\mathbf{b}$, $\mathbf{d})$ were detected by qRT-PCR. The data represent the mean $\pm S D$ for three independent experiments. ${ }^{*} P<0.05,{ }^{* *} P<0.01,{ }^{* * *} P<0.001$

$\mathbf{b}$

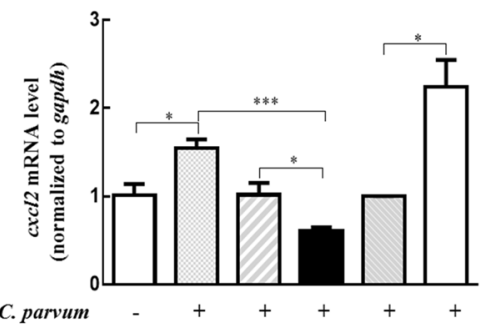

si-control

si-ciRS-7

pcDNA3.1(+)

pcDNA3.1(+)-ciRS-7

d

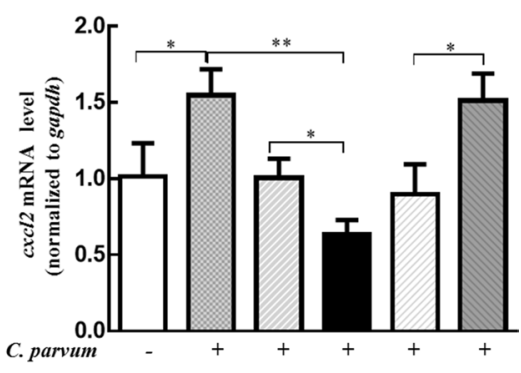

miR-1270 mimics

control inhibitor

1270 inhibitor

\section{a}

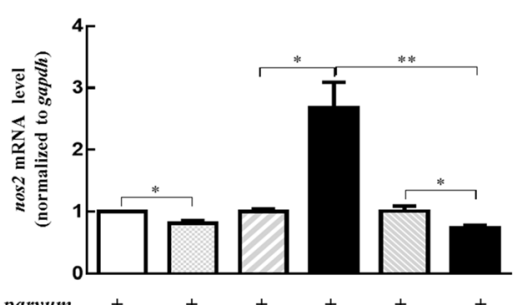

C. parvum

control mimics

miR-1270 mimics

pcDNA3.1 (+)

pcDNA3.1(+)-ciRS-7 
a

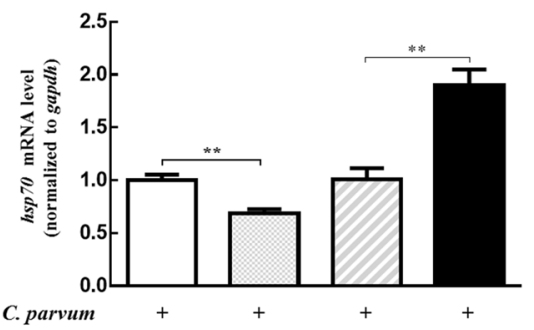

C. parvum

si-control

si-ciRS-7

pcDNA3.1(+)

pcDNA3.1(+)-ciRS-7

$-$

$-$

$+$

c
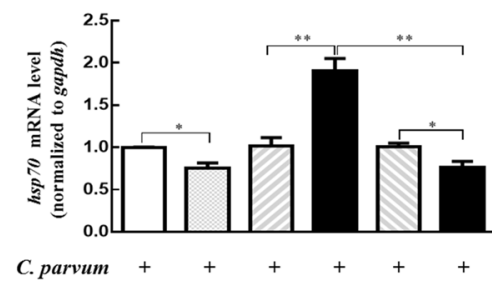

control mimics

miR-1270 mimics

pcDNA3.1(+)

pcDNA3.1(+)-ciRs-7

- - - + + +

Fig. 7 The mRNA levels of C. parvum hsp70 gene in HCT-8 cells. HCT-8 cells were transfected with pcDNA3.1(+)-ciRS-7 plasmid or si-ciRS-7 (a), transfected with miR-1270 mimics or inhibitor (b), and co-transfected with pcDNA3.1(+)-ciRS-7 plasmid and miR-1270 mimics (c), followed by exposure to C. parvum infection for $24 \mathrm{~h}$. The data represent the mean \pm SD for three independent experiments. ${ }^{*} P<0.05$, ${ }^{* *} P<0.01$

(e.g. Alzheimer's disease) [38-41]. In our study, using a C. parvum-infected HCT-8 cell model, ciRS-7 was found to be upregulated at 24 hpi by microarray analysis and at $12-48$ hpi by qRT-PCR tests. Interference of ciRS-7 expression in HCT-8 cells by using siRNA reduced the parasite burden of C. parvum, and transfection of an overexpression (pcDNA3.1(+)-ciRS-7) plasmid into HCT-8 cells elevated the parasite burden. These results indicate that ciRS-7 could promote the propagation of C. parvum in HCT-8 cells through certain functional mechanisms.

To date, several functions of circRNAs have been identified, including acting as miRNA sponges that competitively bind to miRNAs to block miRNA-mRNA interactions, docking in the active sites of RNA-binding proteins (RBPs) to activate these RBPs, modulating mRNA stability, and serving as translation templates $[35,36]$. Among these, sponging miRNA is a well-known regulatory mechanism for circRNAs, although the general feature of circRNAs blocking the miRNA silencing effect remains controversial $[18,42]$. In the present b

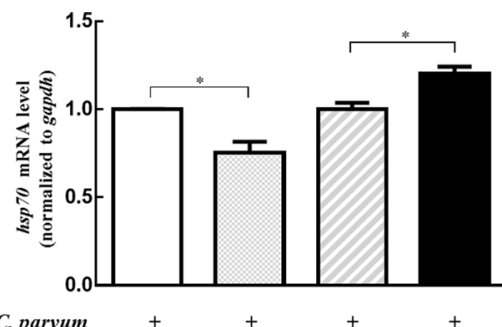

control mimics

miR-1270 mimics

control inhibito

miR-1270 inhibitor study, using the online tool starBase, miR-1270 was predicted to be a potential miRNA target of ciRS-7. Previous studies reported that miR-1270 served as a sponge of ciRS-7 involved in the progression of hepatocellular carcinoma [27] and cisplatin resistance in ovarian cancer and bladder cancer [28, 29]. The luciferase reporter assay validated the interaction between ciRS-7 and miR1270 in HCT-8 cells in our study. C. parvum infection significantly reduced the expression level of miR-1270 in HCT-8 cells. Overexpression of ciRS-7 also decreased miR-1270 in HCT-8 cells infected with C. parvum, while knockdown of ciRS-7 rescued miR-1270 expression in this in vitro infection model, further confirming the sponging effect of ciRS-7 on miR-1270 during C. parvum infection.

Advances in the knowledge of cryptosporidiosis showed that both innate and adaptive immune responses contributed to clear infection and to prevent reinfection of Cryptosporidium, and innate immunity provided a crucially early defense and a key element to activate the adaptive immune system $[7,43]$. Although the initiating 
mechanisms for cellular innate inflammatory responses during Cryptosporidium infection are still poorly understood, the Toll-like receptors (TLRs) (especially TLR2 and TLR4) and the propagation of signaling cascades induced by these TLRs have been reported as important innate elements against Cryptosporidium infection $[43,44]$. C. parvum infection triggered activation of the host NF-kB signaling pathway, a key factor in eliciting inflammatory responses and intracellular survival signals in host epithelial cells during exogenous pathogen infection, but this intrinsic defensive signal was found to be monitored by $C$. parvum to successfully propagate and survive [31, 43]. In our study, we detected continuously high levels of relA mRNA from 12 to 48 hpi in HCT-8 cells infected with C. parvum, and a significantly high protein level was also found at $24 \mathrm{hpi}$. Furthermore, nos 2 and $\operatorname{cxcl} 2$, two important downstream signaling molecules of the NF- $\mathrm{kB}$ signaling pathway, were highly expressed, indicating activation of the NF- $\mathrm{KB}$ signaling pathway in HCT-8 cells infected with C. parvum. Emerging evidence has revealed the significant role of ncRNAs responsible for intricately controlling the innate immunity against Cryptosporidium infection, including miRNAs and the long noncoding RNAs (lncRNAs) [33, 45]. Recently, circRNAs have been identified as key actors in regulating the innate immune responses against viral and bacterial infection [34-36]. We therefore tested the regulatory effect of ciRS-7 on the NF- $\mathrm{kB}$ signaling during infection with C. parvum. Overexpression of ciRS-7 increased the expression of RELA (mRNA and protein) and downstream nos 2 and $c x c l 2$ mRNA levels in infected HCT-8 cells, and vice versa for knockdown of ciRS-7. Considering our finding that ciRS-7 acted as a miRNA sponge for miR-1270 during infection of $C$. parvum, we asked whether ciRS-7 regulated the NF-kB signaling by sponging miR-1270. We confirmed the interaction between miR-1270 and relA by using a luciferase reporter assay, and in a C. parvum-infected HCT-8 cell model, the inhibitory effect of miR-1270 on the expression of relA and downstream nos 2 and $\mathrm{cxcl} 2$ was demonstrated. Notably, cotransfection of miR-1270 mimics and ciRS-7 overexpression vector inhibited the expression of these genes and the burden of C. parvum in HCT- 8 cells. These results suggested that ciRS-7 sponging miR-1270 to regulate the NF- $\mathrm{kB}$ signaling pathway would promote Cryptosporidium infection. However, activation of the NF- $\mathrm{KB}$ signaling pathway would lead to the induction of essential proinflammatory molecules for the host antipathogen response [46, 47], although previous studies have shown that the NF-kB signaling limited the apoptosis of infected cells to facilitate the growth and maturation of C. parvum at the early infection stage $[31,48]$. To address the potential effect of ciRS-7 on host intrinsic immune defense apoptosis during C. parvum infection, we investigated the expression of $\mathrm{Bcl}-2$ (Additional file 11: Figure S6), the central regulator of apoptosis. Upregulation of $\mathrm{Bcl}-2$ was detected from 12 to 48 hpi during C. parvum infection (Additional file 11: Figure S6a). Significantly, overexpression of ciRS-7 further increased the mRNA level of $B c l-2$ at 24 hpi $(P<0.05)$, and silencing of ciRS-7 dramatically decreased the expression of $\mathrm{Bcl}-2$ at $24 \mathrm{hpi}$ $(P<0.05)$ (Additional file 11: Figure S6b). Upregulation of ciRS-7 promoted $\mathrm{Bcl}-2$ expression to inhibit apoptosis, which was also reported in osteoarthritis $(\mathrm{OA})$ model cells [49]. During Crptosporidium infection, apoptosis was increased in HCT-8 cells with siRNA treatment of the $B c l-2$ gene after $C$. parvum infection, and the percentage of infected cells decreased by 1.4 -fold at $24 \mathrm{hpi}$ [50]. These results together with our findings suggested that ciRS-7 may promote the propagation of C. parvum in HCT-8 cells by affecting apoptosis. To further test this hypothesis, infected and neighboring cells in the same cultures should be sorted and evaluated for apoptosis in future studies. Moreover, future studies are needed to explore other functions of the ciRS-7/miR-1270 axis to modulate epithelial cell antiparasite defense during Cryptosporidium infection.

\section{Conclusions}

C. parvum infection altered the expression profiles of circRNAs in HCT-8 cells. Our findings suggested that the ciRS-7/miR-1270 axis may promote the propagation of C. parvum in vitro by activating the host cell NF- $\mathrm{kB}$ signaling pathway and providing a fundamental basis to develop effective strategies against cryptosporidiosis. Certainly, several limitations exist in our work. For example, previous studies showed that $C$. parvum suppressed apoptosis of directly infected cells by activating the NF- $\mathrm{kB}$ signaling in these cells, and it is unclear whether there is an association between the ciRS-7/miR1270 axis and directly infected cell apoptosis. Do other sponging miRNAs of ciRS-7 exist? Is the regulatory effect of the ciRS-7/miR-1270 axis in vivo the same? These scientific questions should be investigated in future studies to comprehensively explain the role of circRNAs during Cryptosporidium infection.

\footnotetext{
Abbreviations

HCT-8 cells: HCT-8 human ileocecal adenocarcinoma cells; qRT-PCR: Quantitative real-time polymerase chain reaction; siRNA: Small interfering RNA; NF-KB: Nuclear factor kappa-light-chain-enhancer of activated B cells; PBS: Phosphate buffered saline; TLRs: Toll like receptors; SDS-PAGE: Sodium dodecyl sulfate polyacrylamide gel electrophoresis; PVDF: Polyvinylidene difluoride; ECL: Enhanced chemiluminescence; WT: Wild-type; MUT: Mutant; DE: Differentially expressed.
} 


\section{Supplementary Information}

The online version contains supplementary material available at https://doi. org/10.1186/s13071-021-04739-w.

Additional file 1: Table S1. Primers for qRT-PCR used in this study (DOCX $16 \mathrm{~KB})$

Additional file 2: Table S2. Sequences of siRNA and miRNA mimics and inhibitors used in this study (DOCX 15 KB)

Additional file 3: Table S3. Significantly differentially expressed circRNAs between C. parvum-infected and noninfected HCT-8 cells (XLSX 95 KB)

Additional file 4: Table $\mathbf{S 4}$. $\mathrm{GO}$ analysis of genes producing DE circRNAs (XLSX 386 KB)

Additional file 5: Table S5. KEGG pathway analysis of genes producing DE circRNAs (XLSX 75 KB)

Additional file 6: Figure $\mathbf{S 1}$. The top 30 significantly enriched terms in GO analysis of genes producing DE circRNAs. Blue bars represent biological process terms. Red bars represent molecular function terms (TIF 2564 KB)

Additional file 7: Figure S2. The top ten significantly enriched terms in KEGG pathway analysis of genes producing DE circRNAs (TIF $1351 \mathrm{~KB}$ )

Additional file 8: Figure S3. The regulatory network of ciRS-7 and its potential sponging miRNAs. The circular red nodes represent circRNAs, and the circular green nodes represent miRNAs (TIF 602 KB)

Additional file 9: Figure S4. ciRS-7 and miR-1270 were successfully inhibited or overexpressed in HCT-8 cells. HCT-8 cells were transfected with four siRNAs targeting ciRS-7 (a), pcDNA3.1(+)-ciRS-7 plasmid (b), miR-1270 inhibitor (c), and miR-1270 mimics (d) for $24 \mathrm{~h}$, and the expression levels of ciRS-7 or miR-1270 were analyzed by qRT-PCR. The data represent the mean $\pm S D$ of three independent experiments. ${ }^{*} P<0.05$, ${ }^{*} P<0.01$ (TIF $332 \mathrm{~KB})$

Additional file 10: Figure S5. The expression of Crytposporidium hsp70 in HCT-8 cells infected with C. parvum. a. The Ct values of Crytposporidium hsp70 for each HCT-8 cell sample with (O1-O3) or without (C1-C3) C. parvum infections obtained from qRT-PCR assays. b. Agarose gels of qRT-PCR assays. Lane M represents DL500 DNA Marker (TIF 379 KB)

Additional file 11: Figure S6. BCl-2 expression in $\mathrm{HCT}-8$ cells following $C$. parvum infection. a Time-dependent expression of $B C-2$ mRNA levels in HCT-8 cells during C. parvum infection. b BCl-2 mRNA levels in HCT-8 cells transfected with pcDNA3.1(+)-ciRS-7 plasmid or si-ciRS-7 and exposed to C. parvum infection for $24 \mathrm{~h}$. The data represent the mean \pm SD for three independent experiments. ${ }^{*} \mathrm{P}<0.05,{ }^{* *} \mathrm{P}<0.01$ (TIF 299 KB)

\section{Authors' contributions}

GHZ conceived and designed the experiments. YLY, TLL, XTW, QY, XMW, YXW, $X Y$, and JKS conducted the experiments and performed mathematical analyses. YLY and GHZ wrote the paper. GHZ designed and supervised the work. All authors reviewed and approved the final manuscript.

\section{Funding}

This study was supported by the grants from the National Natural Science Foundation of China (grant no. 32072890), The Innovation Support Plan of Shaanxi Province (grant no. 2021TD-31), the Open Funds of the State Key Laboratory of Veterinary Aetiological Biology, Lanzhou Veterinary Research Institute, Chinese Academy of Agricultural Sciences (grant no. SKLVEB2020KFKT015), and National Key Research and Development Program of China (grant no. 2017YFD0501305).

\section{Availability of data and materials}

All datasets generated for this study are included in the article/Supplementary Information.

\section{Declarations}

Ethics approval and consent to participate

All experimental procedures in the present research were conducted strictly in accordance with the Guide for the Care and Use of Laboratory Animals of the Ministry of Health, China, and all experimental procedures were reviewed and approved by the Research Ethics Committee of Northwest A\&F University, Yangling, China.

\section{Consent for publication}

Not applicable.

\section{Competing interests}

The authors declare no competing of interest.

\section{Author details}

${ }^{1}$ Department of Parasitology, College of Veterinary Medicine, Northwest A\&F University, Yangling, China. ${ }^{2}$ State Key Laboratory of Veterinary Etiological Biology, Key Laboratory of Veterinary Parasitology of Gansu Province, Lanzhou Veterinary Research Institute, Chinese Academy of Agricultural Sciences, Lanzhou, China.

Received: 24 February 2021 Accepted: 21 April 2021

Published online: 06 May 2021

\section{References}

1. GBD. Global, regional, and national age-sex specific mortality for 264 causes of death, 1980-2016: a systematic analysis for the Global Burden of Disease Study 2016. Lancet. 2017;390:1151-210.

2. Ryan U, Hijjawi N, Xiao L. Foodborne cryptosporidiosis. Int J Parasitol. 2018:48:1-12.

3. Khali IA, Troeger C, Rao PC, Blacker BF, Brown A, Brewer TG, et al. Morbidity, mortality, and long-term consequences associated with diarrhoea from Cryptosporidium infection in children younger than 5 years: a metaanalyses study. Lancet Glob Health. 2018;6:e758-68.

4. Zahedi A, Ryan U. Cryptosporidium — an update with an emphasis on foodborne and waterborne transmission. Res Vet Sci. 2020;132:500-12.

5. Efstratiou A, Ongerth JE, Karanis P. Waterborne transmission of protozoan parasites: review of worldwide outbreaks — an update 2011-2016. Water Res. 2017;114:14-22.

6. Xiao L, Feng Y. Molecular epidemiologic tools for waterborne pathogens Cryptosporidium spp. and Giardia duodenalis. Food Waterborne Parasitol. 2017;8-9:14-32.

7. Checkley W, White AC Jr, Jaganath D, Arrowood MJ, Chalmers RM, Chen $X M$, et al. A review of the global burden, novel diagnostics, therapeutics, and vaccine targets for Cryptosporidium. Lancet Infect Dis. 2015;15:85-94.

8. Relat RMB, O'Connor RM. Cryptosporidium: host and parasite transcriptome in infection. Curr Opin Microbiol. 2020;58:138-45.

9. Wright MW, Bruford EA. Naming "junk": human non-protein coding RNA (ncRNA) gene nomenclature. Hum Genomics. 2011:5:90-8.

10. Tabatabaeian H, Peiling Yang S, Tay Y. Non-coding RNAs: uncharted mediators of thyroid cancer pathogenesis. Cancers (Basel). 2020;12:E3264.

11. Chen LL. The biogenesis and emerging roles of circular RNAs. Nat Rev Mol Cell Biol. 2016:17:205-11.

12. Ma Y, Xu Y, Zhang J, Zheng L. Biogenesis and functions of circular RNAs and their role in diseases of the female reproductive system. Reprod Biol Endocrinol. 2020;18:104

13. Guo R, Chen D, Chen H, Xiong C, Zheng Y, Hou C, et al. Genome-wide identification of circular RNAs in fungal parasite nosema ceranae. Curr Microbiol. 2018;75:1655-60.

14. Ma X, Zhao X, Zhang Z, Guo J, Guan L, Li J, et al. Differentially expressed non-coding RNAs induced by transmissible gastroenteritis virus potentially regulate inflammation and NF-KB pathway in porcine intestinal epithelial cell line. BMC Genomics. 2018;19:747.

15. Yi Z, Gao K, Li R, Fu Y. Dysregulated circRNAs in plasma from active tuberculosis patients. J Cell Mol Med. 2018:22:4076-84. 
16. Chen J, Wang H, Jin L, Wang L, Huang X, Chen W, et al. Profile analysis of circRNAs induced by porcine endemic diarrhoea virus infection in porcine intestinal epithelial cells. Virology. 2019;527:169-79.

17. Liu Y, Hu C, Sun Y, Wu H, Chen X, Liu Q. Identification of differentially expressed circular RNAs in HeLa cells infected with Chlamydia trachomatis. Pathog Dis. 2019;77:ftz062

18. Fan XC, Liu TL, Wang Y, Wu XM, Wang YX, Lai P, et al. Genome-wide analysis of differentially expressed profiles of mRNAs, IncRNAs and circRNAs in chickens during Eimeria necatrix infection. Parasit Vectors. 2020;13:167.

19. Ren GJ, Fan XC, Liu TL, Wang SS, Zhao GH. Genome-wide analysis of differentially expressed profiles of mRNAs, IncRNAs and circRNAs during Cryptosporidium baileyi infection. BMC Genomics. 2018;19:356.

20. Wang R, Zhang L, Axén C, Bjorkman C, Jian F, Amer S, et al. Cryptosporidium parvum IId family: clonal population and dispersal from Western Asia to other geographical regions. Sci Rep. 2014;4:4208.

21. Liu TL, Fan XC, Li YH, Yuan YJ, Yin YL, Wang XT, et al. Expression profiles of mRNA and IncRNA in HCT-8 cells infected with Cryptosporidium parvum Ild subtype. Front Microbiol. 2018;9:1409.

22. Li $X$, Zheng $Y$, Zheng $Y$, Huang $Y$, Zhang $Y$, Jia L, et al. Circular RNA CDR1as regulates osteoblastic differentiation of periodontal ligament stem cells via the miR-7/GDF5/SMAD and p38 MAPK signaling pathway. Stem Cell Res Ther. 2018:9:232

23. Li P, Yang $X$, Yuan W, Yang C, Zhang X, Han J, et al. CircRNA-Cdr1as exerts anti-oncogenic functions in bladder cancer by sponging microRNA-135a. Cell Physiol Biochem. 2018;46:1606-16.

24. Chen H, Mao M, Jiang J, Zhu D, Li P. Circular RNA CDR1as acts as a sponge of miR-135b-5p to suppress ovarian cancer progression. Onco Targets Ther. 2019;12:3869-79.

25. Li JH, Liu S, Zhou H, Qu LH, Yang JH. starBase v2.0: decoding miRNAceRNA, miRNA-ncRNA and protein-RNA interaction networks from largescale CLIP-Seq data. Nucleic Acids Res. 2014;42:D92-7.

26. Shannon P, Markiel A, Ozier O, Baliga NS, Wang JT, Ramage D, et al. Cytoscape: a software environment for integrated models of biomolecular interaction networks. Genome Res. 2003;13:2498-504.

27. Su Y, Lv X, Yin W, Zhou L, Hu Y, Zhou A, et al. CircRNA Cdr 1 as functions as a competitive endogenous RNA to promote hepatocellular carcinoma progression. Aging (Albany NY). 2019;11:8182-203.

28. Zhao Z, Ji M, Wang Q, He N, Li Y. Circular RNA Cdr1as upregulates SCAl to suppress cisplatin resistance in ovarian cancer via miR-1270 suppression. Mol Ther Nucleic Acids. 2019;18:24-33

29. Yuan W, Zhou R, Wang J, Han J, Yang X, Yu H, et al. Circular RNA Cdr1as sensitizes bladder cancer to cisplatin by upregulating APAF1 expression through miR-1270 inhibition. Mol Oncol. 2019;13:1559-76.

30. Lu TX, Rothenberg ME. MicroRNA. J Allergy Clin Immunol. 2018;141:1202-7.

31. Chen XM, Levine SA, Splinter PL, Tietz PS, Ganong AL, Jobin C, et al. Cryptosporidium parvum activates nuclear factor kappaB in biliary epithelia preventing epithelial cell apoptosis. Gastroenterology. 2001;120:1774-83.

32. Cao M, Zhang L, Wang JH, Zeng H, Peng Y, Zou J, et al. Identifying circRNA-associated-ceRNA networks in retinal neovascularization in mice. Int J Med Sci. 2019;16:1356-65.

33. Li M, Gong AY, Zhang XT, Wang Y, Mathy NW, Martins GA, et al. Induction of a long noncoding RNA transcript, NR_045064, promotes defense gene transcription and facilitates intestinal epithelial cell responses against Cryptosporidium infection. J Immunol. 2018;201:3630-4.
34. Li Z, Cheng Y, Wu F, Wu L, Cao H, Wang Q, et al. The emerging landscape of circular RNAs in immunity: breakthroughs and challenges. Biomark Res. 2020:8:25.

35. Li I, Chen YG. Emerging roles of circular RNAs in innate immunity. Curr Opin Immunol. 2020;68:107-15.

36. Xie R, Zhang Y, Zhang J, Li J, Zhou X. The role of circular RNAs in immunerelated diseases. Front Immunol. 2020;11:545.

37. Zhou CX, Ai K, Huang CQ, Guo JJ, Cong H, He SY, et al. miRNA and circRNA expression patterns in mouse brain during toxoplasmosis development. BMC Genomics. 2020;21:46.

38. Azari H, Mousavi P, Karimi E, Sadri F, Zarei M, Rafat M, et al. The expanding role of CDR1-AS in the regulation and development of cancer and human diseases. J Cell Physiol. 2021;236:771-90.

39. Zou Y, Zheng S, Deng X, Yang A, Kong Y, Kohansal M, et al. Diagnostic and prognostic value of circular RNA CDR1as/ciRS-7 for solid tumours: a systematic review and meta-analysis. J Cell Mol Med. 2020;24:9507-17.

40. Kumar L, Haque R, Baghel T, Nazir A. Circular RNAs: the emerging class of non-coding RNAs and their potential role in human neurodegenerative diseases. Mol Neurobiol. 2017;54:7224-34.

41. Akhter R. Circular RNA and Alzheimer's disease. Adv Exp Med Biol. 2018;1087:239-43.

42. Hansen TB, Jensen TI, Clausen BH, Bramsen JB, Finsen B, Damgaard CK, et al. Natural RNA circles function as efficient microRNA sponges. Nature. 2013:495:384-8.

43. Laurent $F$, Lacroix-Lamandé $S$. Innate immune responses play a key role in controlling infection of the intestinal epithelium by Cryptosporidium. Int J Parasitol. 2017:47:711-21.

44. McDonald V, Korbel DS, Barakat FM, Choudhry N, Petry F. Innate immune responses against Cryptosporidium parvum infection. Parasite Immunol. 2013;35:55-64.

45. Zhou R, Feng Y, Chen XM. Non-coding RNAs in epithelial immunity to Cryptosporidium infection. Parasitology. 2014;141:1233-43.

46. Chen XM, O'Hara SP, Nelson JB, Splinter PL, Small AJ, Tietz PS, et al. Multiple TLRs are expressed in human cholangiocytes and mediate host epithelial defense responses to Cryptosporidium parvum via activation of NF-kappaB. J Immunol. 2005;175:7447-56

47. Petry F, Jakobi V, Tessema TS. Host immune response to Cryptosporidium parvum infection. Exp Parasitol. 2010;126:304-9.

48. McCole DF, Eckmann L, Laurent F, Kagnoff MF. Intestinal epithelial cell apoptosis following Cryptosporidium parvum infection. Infect Immun. 2000;68(1710-3):49.

49. Zhou $X$, Jiang L, Fan G, Yang H, Wu L, Huang Y, et al. Role of the ciRS-7/ miR-7 axis in the regulation of proliferation, apoptosis and inflammation of chondrocytes induced by IL-1ß. Int Immunopharmacol. 2019;71:233-40.

50. Liu J, Deng M, Lancto CA, Abrahamsen MS, Rutherford MS, Enomoto S. Biphasic modulation of apoptotic pathways in Cryptosporidium parvuminfected human intestinal epithelial cells. Infect Immun. 2009;77:837-49.

\section{Publisher's Note}

Springer Nature remains neutral with regard to jurisdictional claims in published maps and institutional affiliations.

Ready to submit your research? Choose BMC and benefit from:

- fast, convenient online submission

- thorough peer review by experienced researchers in your field

- rapid publication on acceptance

- support for research data, including large and complex data types

- gold Open Access which fosters wider collaboration and increased citations

- maximum visibility for your research: over 100M website views per year

At BMC, research is always in progress.

Learn more biomedcentral.com/submissions 